\title{
Microleakage of Four Dental Cements in Metal Ceramic Restorations With Open Margins
}

\author{
Reza Eftekhar Ashtiani, ${ }^{1}$ Babak Farzaneh, ${ }^{2}$ Mohadese Azarsina, ${ }^{3}$ Farzad Aghdashi, ${ }^{4, *}$ Nima \\ Dehghani, ${ }^{4}$ Aisooda Afshari, ${ }^{5}$ and Minu Mahshid ${ }^{6}$ \\ ${ }^{1}$ Department of Dental Technology, School of Dentistry, Shahid Beheshti University of Medical Sciences, Tehran, IR Iran \\ 2 Endodontics Research Center, AJA University of Medical Sciences, Tehran, IR Iran \\ ${ }^{3}$ Department of Operative Dentistry, Dental School, International Branch of Shahid Beheshti University of Medical Sciences, Tehran, IR Iran \\ ${ }_{5}^{4}$ Department of Oral and Maxillofacial Surgery, Dental School, Taleghani Hospital, Shahid Beheshti University of Medical Sciences, Tehran, IR Iran \\ 5 Shiraz University of Medical Sciences, Shiraz, IR Iran \\ 6 Shiraz University of Medical Sciences, Shiraz, IR Iran
${ }_{\text {Department of Prosthetic, Dental School, Shahid Beheshti University of Medical Sciences, Tehran, IR Iran }}$ \\ *Corresponding Author: Farzad Aghdashi, Department of Oral and Maxillofacial Surgery, Dental School, Taleghani Hospital, Shahid Beheshti University of Medical Sciences, Tehran, \\ IR Iran. Tel: +98-9126214250, E-mail: Fal2zad@gmail.com
}

Received: April 22, 2014; Revised: August 13, 2014; Accepted: September 9, 2014

Background: Fixed prosthodontics is a routine dental treatment and microleakage is a major cause of its failure.

Objectives: The aim of this study was to assess the marginal microleakage of four cements in metal ceramic restorations with adapted and open margins.

Materials and Methods: Sixty sound human premolars were selected for this experimental study performed in Tehran, Iran and prepared for full-crown restorations. Wax patterns were formed leaving a $300 \mu \mathrm{m}$ gap on one of the proximal margins. The crowns were cast and the samples were randomly divided into four groups based on the cement used. Copings were cemented using zinc phosphate cement(Fleck), Fuji Plus resin-modified glass ionomer, Panavia F2.0 resin cement, or G-Cem resin cement, according to the manufacturers' instructions. Samples were immersed in 2\% methylene blue solution.After 24 hours, dye penetration was assessed under a stereomicroscope and analyzed using the respective software. Data were analyzed using ANOVA, paired t-tests, and Kruskal-Wallis, Wilcoxon, and Mann-Whitney tests.

Results: The least microleakage occurred in the Panavia F2.0 group (closed margin, $0.18 \mathrm{~mm}$; open margin, $0.64 \mathrm{~mm}$ ) and the maximum was observed in the Fleck group (closed margin, $1.92 \mathrm{~mm}$; open margin, $3.32 \mathrm{~mm}$ ). The Fleck group displayed significantly more microleakage compared to the Fuji Plus and Panavia F2.0 groups $(\mathrm{P}<0.001)$ in both closed and open margins. In open margins, differences in microleakage between the Fuji Plus and G-Cem as well as between the G-Cem and Panavia F2.0 groups were significant $(\mathrm{P}<0.001)$. In closed margins, only the G-Cem group displayed significantly more microleakage as compared to the Panavia F2.0 group $(\mathrm{P}<0.05)$. Paired t-test results showed significantly more microleakage in open margins compared to closed margins, except in the Fuji Plus group $(\mathrm{P}=0.539)$.

Conclusions: Fuji Plus cement exhibited better sealing ability in closed and open margins compared to G-Cem and Fleck cements. When using G-Cem and Fleck cements for full metal ceramic restorations, clinicians should try to minimize marginal gaps in order to reduce restoration failure. In situations where there are doubts about perfect marginal adaptation, the use of Fuji Plus cement may be helpful.

Keywords: Dental Leakage; Metal Ceramic Restorations; Tooth

\section{Background}

Fixed prosthodontics is a commonly used dental treatment that restores and preserves oral tissues. Marginal gaps at the tooth-restoration interface are an inevitable component of any fixed restoration and need to be sealed effectively. Luting cements are used to seal gaps and preserve the tooth from microbial invasion (1). Failure of cements to seal gaps may result in an inflammatory response in the pulp and subsequent pulpal necrosis, which compromises the longevity of restorations $(1,2)$. Microleakage is indicated by post-treatment hypersensitivity, chronic tooth hypersensitivity, and marginal discoloration around the restoration (3). Water-based cements have been used traditionally as space fillers be- tween the tooth and restoration. However, due to their high solubility in oral fluids, the sealing ability of these cements is highly dependent on the accuracy of the restoration margins (4). The largest acceptable value for a tooth/restoration gap ranges between 100 - $500 \mu \mathrm{m}$ (5-7). In order to overcome the likely problems associated with marginal gaps, clinicians should follow technical guidelines accurately and only apply the most high-quality materials when constructing prostheses in order to achieve maximum marginal compatibility. In addition, the use of cements with satisfactory sealing ability is crucial to minimize marginal leakage. Although different types of cements have been introduced in dentistry, glass 
ionomers and resin-based cements have been shown to exhibit the most favorable results due to the release of fluoride ions, which establishes an additional micromechanical bond with the tooth $(4,8)$.

Biocompatibility, sealing ability, and caries prevention are the important characteristics of an ideal cement. Microbial microleakage may be the most important factor influencing the long term success of a restoration (3). Studies have shown that resin-modified glass ionomers and cements with bonding capacity to the tooth exhibit less microleakage compared to conventional cements (e.g. zinc phosphate cements) $(1,4,9,10)$. Numerous studies have evaluated the sealing ability of different cements in different restorations, on different tooth types, with various surface treatments, convergence angles, and numbers of samples (11).

\section{Objectives}

This study aimed to compare the microleakage of four luting cements in metal ceramic restorations with open and closed margins.

\section{Materials and Methods}

An in vitro experimental study was designed to assess the microleakage of four different cements. This study was performed in the Shahid Beheshti Dental School, Tehran, Iran. Sixty sound human first premolar teeth, extracted for orthodontic purposes, were obtained and stored in $1 \%$ chloramine solution, which is known to have no effect on dentinal permeability and the bond strength of cement. The teeth were caries free, with no cracks, craze lines, or cervical lesions. We collected the extracted teeth after informed consent was obtained from the patients, according to the ethical committee rules of Shahid Beheshti medical university. All teeth were prepared for a full coverage crown in the same way by one investigator. The occlusal and axial surfaces were reduced to $1.5 \mathrm{~mm}$ and $1 \mathrm{~mm}$, respectively, with a $135^{\circ}$ sloping shoulder finish line in the dentin. A groove was created on the buccal cusp of the teeth to facilitate placement and adaptation of the crown. The required time for each tooth preparation was 10 minutes, and burs (D and Z, Germany) were replaced after every third tooth preparation. Impressions were obtained from each tooth using polyether Impregum impression material (ESPE, Germany) and casts were poured with type III die stone (Die Stone, USA). Dies were prepared and covered with two layers of die spacer (Bego, Germany) at a 1-mm distance from the margin of the die. Copings were then formed using inlay wax, leaving a 300- $\mu \mathrm{m}$ space ( $2 \mathrm{~mm}$ long buccolingually and shoulder wide mesiodistally) on one of the proximal surfaces. This space was created using a piece of paper with the respective dimensions, the thickness of which was verified accurately using a micrometer and stereomicroscope (Figure 1). Sprues were attached identically on the buccal cusp of each specimen and investing was performed subsequently using investment phosphate bond (Bego-Begovest,
Germany), by placing four teeth in each cylinder. Casting and burnout were carried out with base metal (Verabond, USA) and Begos-Nautilus (Castomatte), respectively. Each metal coping was seated on the respective die and checked for full compatibility with a fit checker (GC, Japan). The gap between the metal coping and the tooth was measured carefully and those with $>350 \mu \mathrm{m}$ and $<250 \mu \mathrm{m}$ of space at the intended proximal margin of the restoration were subjected to re-impression and casting (Figure 2). Copings were subsequently coded and the specimens were randomly divided into four groups. Copings were cemented onto the respective tooth using zinc phosphate cement (Fleck, USA), Fuji Plus resin-modified glass ionomer (GC, Japan), Panavia F2.0 resin cement (Kurrary, Japan), or GCem resin cement (GC, Japan). All copings were cemented according to the manufacturers' instructions and stored in an artificial oral environment $\left(36^{\circ} \mathrm{C}\right)$ for 90 days. After complete setting, the teeth underwent thermal shock cycling (1000 cycles between $5^{\circ} \mathrm{C}$ and $55^{\circ} \mathrm{C}$ for 1 second per cycle). To prevent dye penetration through the apex or dentinal tubules, the teeth were covered with a layer of nail varnish at a distance of $1 \mathrm{~mm}$ from the margin of the coping and subsequently immersed in $2 \%$ methylene blue solution for 24 hours. The samples were then fixed in translucent orthodontic resins and cut mesiodistally to cross the open margin in the buccal area. Each section was observed under a stereomicroscope (Zeiss, Oberkochen, Germany) to assess dye penetration and analyzed using the respective software (Axio Vision 3.1; Zeiss, Oberkochen, Germany) (figure 3) The software was calibrated with original scales and in 10 separate trials indicated only a 50- $\mu \mathrm{m}$ measurement error compared to the control site. All the samples were prepared and tested by a single trained clinician.

All data sets were subjected to normality testing using the Kolmogorov-Smirnov test. Repeated measures ANOVA, paired t-tests, and Kruskal-Wallis, Wilcoxon, and Mann-Whitney tests were used for statistical analysis. The statistical software used was SPSS 16.0.

\section{Results}

Table 1 illustrates the microleakage of the cements in restorations with closed margins. One-way ANOVA revealed a significant difference among the four experimental groups ( $\mathrm{P}<0.001)$. Post-hoc Tukey's tests failed to reveal a significant difference between the mean microleakage of the Panavia F2.0 and Fuji Plus groups, which had the lowest mean microleakage values, or between the mean microleakage of the Fleck and G-Cem groups, which had the highest mean microleakage values. However, the differences between the groups with the least microleakage (i.e. Panavia F2.0 and Fuji Plus) and the groups with the most microleakage (i.e., Fleck and GCem) were shown to be significant. Table 2 summarizes the paired comparison results retrieved from the posthoc Tukey's tests among the experimental groups.

Table 3 illustrates the tubular microleakage in restora- 
tions with open and closed margins.

The Fleck group displayed significantly more tubular microleakage compared to the Fuji Plus and Panavia F2.0 groups $(\mathrm{P}<0.001)$ in both closed and open margins. In open margins, the difference in tubular microleakage between the Fuji Plus and G-Cem groups and between the G-Cem and Panavia F2.0 groups was significant $(\mathrm{P}<$ 0.001). In closed margins, however, only G-Cem cement displayed significantly more tubular microleakage compared to Panavia F2.0 cement $(\mathrm{P}<0.05)$.

The paired t-test results suggested that all groups displayed significantly more marginal microleakage in open margins compared to closed margins, except for the Fuji Plus group $(\mathrm{P}=0.539)$. To compare tubular microleakage between open and closed margins in each group, the Wilcoxon test was utilized and the results failed to prove any significant difference in the Fleck $(\mathrm{P}=0.083)$, Fuji Plus $(\mathrm{P}=0.317)$, and Panavia $\mathrm{F} 2.0(\mathrm{P}=0.318)$ groups. However, open margins displayed significantly more tubular microleakage compared to closed margins in the G-Cem group $(\mathrm{P}<0.05)$.

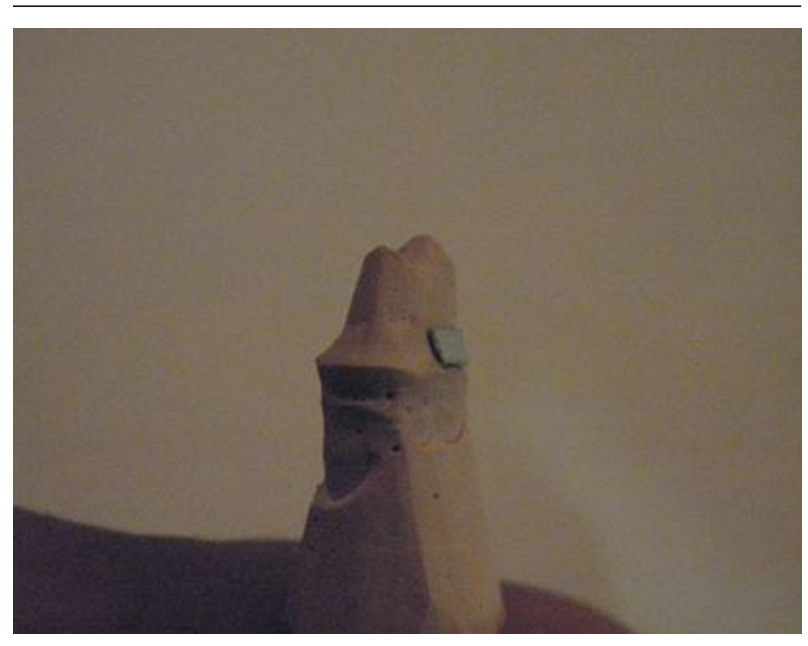

Figure 1. Space Was Created Using a Paper in One Proximal Side of Die

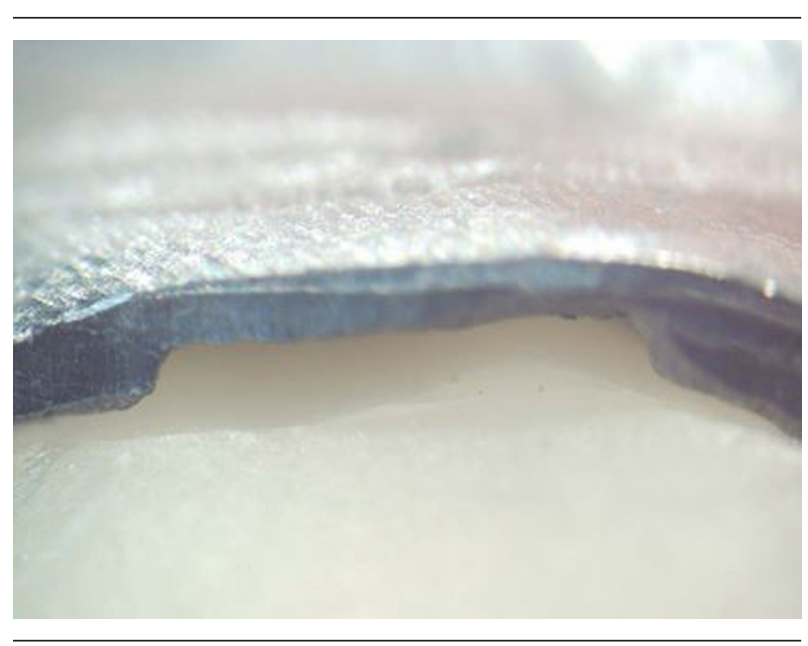

Figure 2. Open Margin Observed Under a Stereomicroscope

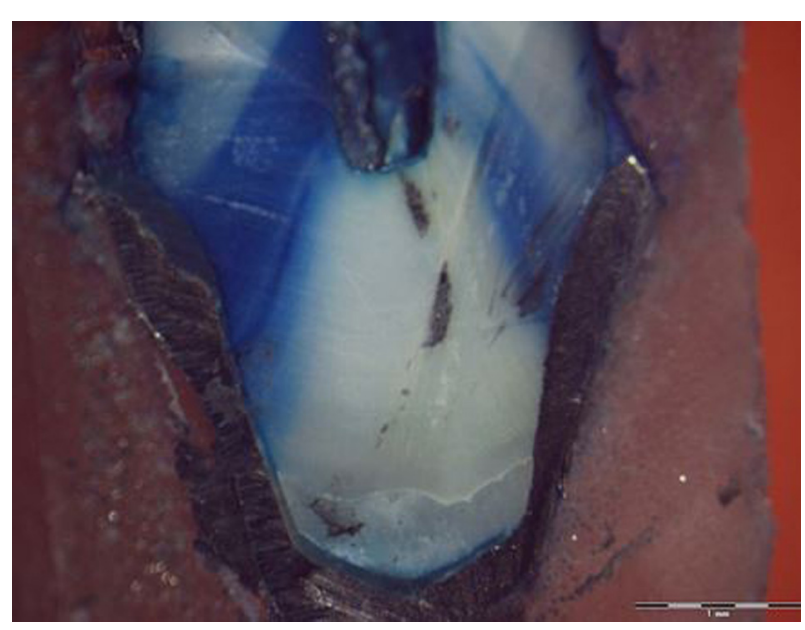

Figure 3. Cross Sectional View of a Tooth and Restoration Showing Dye Penetration

Table 1. Mean and Standard Deviation Values of Microleakage of the Cements in Restorations With Closed Margins (mm)

\begin{tabular}{|c|c|c|}
\hline Cement & $\mathbf{N}$ & Mean \pm SD \\
\hline Fleck ${ }^{\text {a,b }}$ & 15 & $1.92 \pm 1.23$ \\
\hline Fuji Plus a,c & 15 & $0.77 \pm 0.88$ \\
\hline G-Cem c,d & 15 & $1.25 \pm 1.07$ \\
\hline Panavia F2.0 b,d & 15 & $0.18 \pm 0.14$ \\
\hline Total & 60 & $1.03 \pm 1.11$ \\
\hline
\end{tabular}

${ }^{\mathrm{a}}$ Indicate significant difference between Fleck and Fuji Plus $(\mathrm{P}<0.05)$.

$\mathrm{b}$ Indicate significant difference between Fleck and Panavia F2.0 $(\mathrm{P}<0.05)$.

${ }^{\mathrm{C}}$ Indicate significant difference between Fuji Plus and G-Cem $(\mathrm{P}<0.05)$.

$\mathrm{d}$ Indicate significant difference between G-Cem and Panavia F2.0 $(\mathrm{P}<0.05)$.

Table 2. Mean and standard deviation values of microleakage of the cements in restorations with open margins ( $\mathrm{mm}$ )

\begin{tabular}{lcc}
\hline Cement & N & Mean \pm SD \\
\hline Fleck a,b,c & 15 & $3.32 \pm 0.70$ \\
\hline Fuji Plus a,d & 15 & $0.92 \pm 0.53$ \\
\hline G-Cem b,d,e & 15 & $2.08 \pm 1.10$ \\
\hline Panavia F2.0 c,e & 15 & $0.64 \pm 0.78$ \\
\hline Total & 60 & $1.74 \pm 1.32$ \\
\hline
\end{tabular}

a Indicate significant difference between Fleck and Fuji Plus $(\mathrm{P}<0.001)$.

$\mathrm{b}$ Indicate significant difference between Fleck and G-Cem $(\mathrm{P}<0.001)$

${ }^{\mathrm{C}}$ Indicate significant difference between Fleck and Panavia F2.0 $(\mathrm{P}<0.001)$.

$\mathrm{d}$ Indicate significant difference between Fuji Plus and G-Cem $(\mathrm{P}<0.001)$.

e Indicate significant difference between G-Cem and Panavia F2.0 $(\mathrm{P}<0.001)$. 
Eftekhar Ashtiani R et al.

\begin{tabular}{|c|c|c|c|c|c|c|c|}
\hline \multirow[t]{2}{*}{ Cement } & \multicolumn{3}{|c|}{ Closed Margin } & \multicolumn{3}{|c|}{ Open Margin } & \multirow{2}{*}{ Total } \\
\hline & $\mathbf{o}^{\mathbf{b}}$ & $1^{c}$ & $2^{d}$ & $\mathbf{0}$ & 1 & 2 & \\
\hline Fleck & $3(20)$ & $0(0.00)$ & $12(80)$ & $0(0.00)$ & $0(0.00)$ & $15(100)$ & 30 \\
\hline Fuji Plus & $15(100)$ & $0(0.00)$ & $0(0.00)$ & $14(93.3)$ & $1(6.7)$ & $0(0.00)$ & 30 \\
\hline G-Cem & $7(46.7)$ & $1(6.7)$ & $7(46.7)$ & $3(20.0)$ & $1(6.7)$ & $11(73.3)$ & 30 \\
\hline Panavia F2.0 & $15(100)$ & $0(0.00)$ & $0(0.00)$ & $14(93.3)$ & $0(0.00)$ & $1(6.7)$ & 30 \\
\hline Total & $40(66.7)$ & $1(1.7)$ & $19(31.7)$ & $31(51.7)$ & $2(3.3)$ & $27(45.00)$ & 120 \\
\hline
\end{tabular}

a Data are presented as No (\%).

$\mathrm{b}$ No dye penetration into the dentinal tubules.

c Dye penetration half way towards the pulp.

$\mathrm{d}$ Complete dye penetration reaching the pulp.

\section{Discussion}

This study evaluated the sealing ability of different cements (especially self-etch/bond cements) in potential open areas of restoration margins. Panavia F2.0 and Fuji Plus cements displayed more satisfactory results for sealing of potential spaces in closed margins $(<100 \mu \mathrm{m})$ and open margins (> $300 \mu \mathrm{m})$. Minimum solubility, high strength, greater retention, and solid bonding to the tooth structure are among the most important properties of resin cements (11). Different studies have shown less microleakage of resin cements in comparison with other types of cements $(4,8,11)$. However, problems associated with their manipulation and isolation, specifically around the finish line, remain a concern to clinicians and may compromise the longevity of restorations. Recently, manufacturers have introduced a new generation of selfetch/bond cements, which are less sensitive to the technique used in the subgingival margins of restorations. Numerous studies have demonstrated low levels of microleakage with these resins $(12,13)$. Although most previous studies have reported that microleakage does not necessarily correlate with the size of the marginal gap, and the long term success rate of restorations is affected by several other factors (14), the results of the present study indicated a correlation between marginal gap size and microleakage. This result is consistent with the results of the study by Yuksel et al. (15).

The marginal adaptability of restorations is regarded as an important determinant of their long-term success rate (16). In order to eliminate the effect of marginal adaptability as a confounding factor, we investigated the sealing ability of the cements in both closed and open margins. Significant microleakage in the Fleck group was consistent with previous studies $(1,9,10,16)$, which may be attributed to high solubility or failure in establishing a solid bond with the tooth (8-10). In the present study, GCem cement displayed more microleakage as compared to Fuji Plus or Panavia F2.0 cements, which is in contrast to the results of a study performed by Hooshmand et al. who reported more microleakage with Fuji Plus and Panavia F2.0 as compared to the self-adhesive cement tested
(17). This may have been the result of failing to protect the G-Cem cement from the oral environment during the first hour of its polymerization. In addition, the composition of this self-adhesive luting material is more similar to a glass ionomer, as it is less resinous than other resin cements. Therefore, the greater microleakage detected with this cement can be attributed to its higher solubility and monomer leaching during the first hours of polymerization (18, 19). Additionally, polymerization shrinkage of G-Cem may have caused higher stress at the tooth-restoration bond, although further studies are needed to compare the shrinkage of G-Cem with other cements such as Panavia F2.0. On the other hand, Fuji Plus and Panavia F2.0 displayed very low microleakage in the present study, which was consistent with previous reports $(14,20)$.

Although the marginal gaps investigated in the majority of previous studies were within the normal range, some studies have documented the possibility of greater marginal gaps in clinical conditions $(5,7)$. Moreover, the restoration margins in the present study were placed in dentin, which has been shown to display greater microleakage compared to enamel (21).

Inadequate sealing of dentinal tubules may cause pulpal inflammation, which subsequently affects the long term success rate of restorations. No significant correlation was observed between tubular microleakage and the compatibility of the restoration margins in the Fleck, Fuji Plus, and Panavia F2.0 cement groups; however, G-Cem demonstrated significantly better tubular sealing ability in closed margins.

This study was performed in laboratory conditions; more clinical experience is needed to confirm the results in real clinical situations. In this study, only four types of dental cements were evaluated. However, microleakage has not been assessed in non-adapted margins compared with adapted margins in previous studies.

Within the limitations of this study, it was concluded that Panavia F2.0 and Fuji Plus cements exhibited better sealing ability in closed and open margins compared to G-Cem and Fleck cements. When using G-Cem and Fleck cements for full metal ceramic restorations, clinicians should take 
the necessary measures to minimize the marginal gap in order to prevent plaque retention, gingival inflammation, and a less retentive restoration. In situations where there are doubts about perfect marginal adaptation, the use of Panavia F2.0 or Fuji Plus cement may be helpful.

\section{Acknowledgements}

We would like to thank the AJA university of medical sciences for their support of this research.

\section{Authors' Contributions}

Babak Farzaneh, study conception; Reza Eftekhar Ashtiani and Mohadese Azarsina, preparation of the manuscript; Farzad Aghdashi, corresponding author; Nima Dehghani and Aisooda Afshari, data collection and analysis; Minu Mahshid, final proof reading.

\section{Funding/Support}

AJA university of medical sciences, Tehran, Iran.

\section{References}

1. Rosenstiel SF, Land MF, Crispin BJ. Dental luting agents: A review of the current literature. J Prosthet Dent. 1998;80(3):280-301.

2. Mjor IA, Moorhead JE, Dahl JE. Reasons for replacement of restorations in permanent teeth in general dental practice. Int Dent $J$. 2000;50(6):361-6.

3. Larson TD. The clinical significance of marginal fit. Northwest Dent. 2012;91(1):22-9.

4. Hill EE, Lott J. A clinically focused discussion of luting materials. Aust Dent J. 2011;56 Suppl 1:67-76.

5. Wolfart S, Wegner SM, Al-Halabi A, Kern M. Clinical evaluation of marginal fit of a new experimental all-ceramic system before and after cementation. Int J Prosthodont. 2003;16(6):587-92.

6. Kokubo Y, Ohkubo C, Tsumita M, Miyashita A, Vult von Steyern P, Fukushima S. Clinical marginal and internal gaps of Procera AllCeram crowns. J Oral Rehabil. 2005;32(7):526-30.
7. Gemalmaz D, Kukrer D. In vivo and in vitro evaluation of marginal fit of class II ceromer inlays. J Oral Rehabil. 2006;33(6):436-42.

8. Burke FJ. Trends in indirect dentistry: 3. Luting materials. Dent Update. 2005;32(5):251-4-260.

9. Mitchell CA, Abbariki M, Orr JF. The influence of luting cement on the probabilities of survival and modes of failure of cast fullcoverage crowns. Dent Mater. 2000;16(3):198-206.

10. Toman M, Toksavul S, Artunc C, Turkun M, Schmage P, Nergiz I. Influence of luting agent on the microleakage of all-ceramic crowns. J Adhes Dent. 2007;9(1):39-47.

11. Heintze SD. Crown pull-off test (crown retention test) to evaluate the bonding effectiveness of luting agents. Dent Mater. 2010;26(3):193-206

12. Albert FE, El-Mowafy OM. Marginal adaptation and microleakage of Procera AllCeram crowns with four cements. Int J Prosthodont. 2004;17(5):529-35.

13. el-Mowafy $\mathrm{O}$. The use of resin cements in restorative dentistry to overcome retention problems. J Can Dent Assoc. 2001;67(2):97-102.

14. Rossetti PH, do Valle AL, de Carvalho RM, De Goes MF, Pegoraro LF. Correlation between margin fit and microleakage in complete crowns cemented with three luting agents. J Appl Oral Sci. 2008;16(1):64-9.

15. Yuksel E, Zaimoglu A. Influence of marginal fit and cement types on microleakage of all-ceramic crown systems. Braz Oral Res. 2011;25(3):261-6.

16. Lindquist TJ, Connolly J. In vitro microleakage of luting cements and crown foundation material.J Prosthet Dent. 2001;85(3):292-8.

17. Hooshmand T, Mohajerfar M, Keshvad A, Motahhary P. Microleakage and marginal gap of adhesive cements for noble alloy full cast crowns. Oper Dent. 2011;36(3):258-65.

18. Bortolotto T, Guillarme D, Gutemberg D, Veuthey JL, Krejci I. Composite resin vs resin cement for luting of indirect restorations: comparison of solubility and shrinkage behavior. Dent MaterJ. 2013;32(5):834-8.

19. Nakamura T, Wakabayashi K, Kinuta S, Nishida H, Miyamae M, Yatani H. Mechanical properties of new self-adhesive resin-based cement. J Prosthodont Res. 2010;54(2):59-64.

20. Trajtenberg CP, Caram SJ, Kiat-amnuay S. Microleakage of allceramic crowns using self-etching resin luting agents. Oper Dent. 2008;33(4):392-9.

21. Kasraei S, Azarsina M, Majidi S. In vitro comparison of microleakage of posterior resin composites with and without liner using two-step etch-and-rinse and self-etch dentin adhesive systems. Oper Dent. 2011;36(2):213-21. 